\title{
Genome-wide data reveal cryptic diversity and genetic introgression in an Oriental cynopterine fruit bat radiation
}

Balaji Chattopadhyay ${ }^{1,2,3^{*}}$, Kritika M. Garg ${ }^{2,3}$, A. K. Vinoth Kumar ${ }^{1}$, D. Paramanantha Swami Doss ${ }^{1}$, Frank E. Rheindt ${ }^{3}$, Sripathi Kandula' and Uma Ramakrishnan ${ }^{2}$

\begin{abstract}
Background: The Oriental fruit bat genus Cynopterus, with several geographically overlapping species, presents an interesting case study to evaluate the evolutionary significance of coexistence versus isolation. We examined the morphological and genetic variability of congeneric fruit bats Cynopterus sphinx and C. brachyotis using 405 samples from two natural contact zones and 17 allopatric locations in the Indian subcontinent; and investigated the population differentiation patterns, evolutionary history, and the possibility of cryptic diversity in this species pair.

Results: Analysis of microsatellites, cytochrome $b$ gene sequences, and restriction digestion based genome-wide data revealed that C. sphinx and C. brachyotis do not hybridize in contact zones. However, cytochrome $b$ gene sequences and genome-wide SNP data helped uncover a cryptic, hitherto unrecognized cynopterine lineage in northeastern India coexisting with C. sphinx. Further analyses of shared variation of SNPs using Patterson's D statistics suggest introgression between this lineage and C. sphinx. Multivariate analyses of morphology using genetically classified grouping confirmed substantial morphological overlap between C. sphinx and C. brachyotis, specifically in the high elevation contact zones in southern India.

Conclusion: Our results uncover novel diversity and detect a pattern of genetic introgression in a cryptic radiation of bats, demonstrating the complicated nature of lineage diversification in this poorly understood taxonomic group. Our results highlight the importance of genome-wide data to study evolutionary processes of morphologically similar species pairs. Our approach represents a significant step forward in evolutionary research on young radiations of non-model species that may retain the ability of interspecific gene flow.
\end{abstract}

Keywords: Gene flow, SNPs, ddRAD, Cynopterus sphinx, Cynopterus brachyotis

\section{Background}

Understanding and identifying cryptic diversity directly informs conservation and plays a crucial role in the formulation of management decisions $[1,2]$. This is particularly important for species pairs that show geographical as well as morphological overlap [3-6], wherein taxonomic identification remains a difficulty and evolutionary processes go undetected [5-7]. In such cases a composite approach of supplementing morphological identification with genetic classification considerably improves species identification,

\footnotetext{
* Correspondence: balaji.chattopadhyay@gmail.com

${ }^{1}$ School of Biological Sciences, Madurai Kamaraj University, Madurai, India

${ }^{2}$ Ecology and Evolution, National Centre for Biological Science, TIFR,

Bangalore, India

Full list of author information is available at the end of the article
}

detects cryptic diversity, thereby providing a better inventory of natural history and biodiversity [2, 5-9]. Such an approach also acts as a primer to understand evolutionary trajectories of codistributed species pairs and behavioral as well as ecological contingencies of coexistence.

Restriction digestion based genome-wide data often retrieve thousands to hundreds of thousands of loci and in recent times have provided unparalleled resolution towards the understanding of genetic diversity, gene flow and evolutionary history, specifically of non model organisms. For example, using a few million base pairs of sequence data, Wagner et al. [10] were able to differentiate between various lineages of Lake Victoria cichlids, which diversified only 15,000 years ago. Other genomic scans 
have revealed the importance of transposable elements in maintaining different butterfly races [11], parallel phenotypic evolution in sticklebacks involving similar regions of the genome [12], rare introgression [13] and inbreeding [14]. Genome-wide data also shows great promise towards the discovery and understanding of cryptic diversity, and evolutionary studies of non-model organisms $[2,9,15]$.

Old world fruit bats of the genus Cynopterus present an interesting natural system to study evolutionary dynamics of codistributed species pairs. The genus Cynopterus has undergone a recent, relatively rapid radiation giving rise to species complexes whose phylogeny remains unresolved $[3,16]$. Many of these lineages share broad zones of coexistence across south and southeast Asia [3]. Most cynopterine species show considerable overlap in niche space and morphology, as diets are simple and non-specialized [17]. Species level identification of cynopterine fruit bats remains a problem, especially in contact zones [17-20] and genetic diagnosis remains a necessity, especially in the absence of extensive collections and detailed morphological information from many regions.

In the present study, we assessed morphological and genetic diversity of two congeneric cynopterine fruit bats, Cynopterus sphinx and C. brachyotis. These species are closely related [3] and show broad morphological overlap in areas of coexistence $[3,19,20]$. We characterized zones of overlap between these two species using morphological, population genetic and phylogenetic analyses. We examined morphological variation based on species-specific phenotypic characters (following $[18,20]$ ), obtained genetic classification of our dataset without a priori assumption of group membership using autosomal microsatellite markers and up to 10,000 SNPs derived from genomewide data (double digest restriction site associated DNA sequencing, ddRADseq following [21]) and applied mitochondrial cytochrome $b$ (cytb) gene sequences along with upto $\sim 700,000 \mathrm{bp}$ of sequence data derived from ddRADseq to reconstruct the species phylogeny. Using our genome-wide dataset, we also tested for the effect of missing data and an increase in the number of loci in genetic assignment and phylogeny reconstruction. We further used classifications based on genetic markers to assess the extent of morphological overlap between both species and generated classification functions for morphological variables that can be used for field identification of cynopterine bats in India.

We document the presence of a cryptic cynopterine lineage and reveal introgression between deeply diverged species-level cynopterine lineages in northeastern India. We also demonstrate that genome-wide data spanning thousands of loci are robust to the effects of missing data. Lastly, our phenotypic examinations have failed to come up with diagnostic morphological traits for species level classification in the contact zones and we suggest that genetic data, specifically genome-wide SNPs should be used for species identification.

\section{Methods \\ Ethics statement}

This study and sampling protocols were approved by and were in accordance with the institutional ethics committees (Internal Research Review Board (IRB), Ethical Clearance (EC), Biosafety and Animal Welfare committee approval to Balaji Chattopadhyay dated 21-11-2005 Madurai Kamaraj University and Institutional Animal Ethics Committee (IACE) to Uma Ramakrishnan (UR-3/2009), National Centre for Biological Sciences). The study species are not endangered and are classified under Least Concern category in the International Union for Conservation of Nature (IUCN) red list.

\section{Sampling}

We sampled bats between August 2005 and February 2012 at 19 locations across India (Fig. 1 and Additional file 1: Table S1). The study species are considered as vermin under the Indian Wildlife protection Act (1972). These are not protected species and sampling them outside reserve forest limits does not require any permission. We performed sampling following forest department permit (WL5/44926/2010, dated: 11.03.2011) within reserve forest limits (locations: 2 and 7, Additional file 1: Table S1). All other sampling locations were outside reserve forest limits and we obtained oral permissions from owners whenever we sampled in private areas.

We captured bats either in hoop nets at their day roost or in mist nets (Avinet Inc., USA) at foraging grounds after nightfall. We classified individuals as juveniles or adults based on the extent of tooth wear of the upper canines, pelage colouration and ossification of epiphyseal bones [20, 22-24]. We used only adult individuals for morphometric analyses, whereas all individuals were subjected to molecular analyses.

We measured lengths of the forearm, tibia and ear of each bat using a dial caliper (Avinet Inc, USA). We also used ear margins as a categorical variable by coding it as distinct, faint or absent. We initially identified bats in the field as C. sphinx or C. brachyotis following Bates and Harrison [18] and Storz and Kunz [20]. However, we also frequently observed bats with a long forearm length (characteristic of C. sphinx), but with either pale or no ear margin (characteristic of C. brachyotis). Because of this general lack of consensus regarding morphological characters we used the following scheme for field identification based on our prior field experience in the southern Western Ghats. We gave major weightage to the presence of an ear margin, assigning to C. sphinx all bats with a prominent ear margin and to C. brachyotis those with no margin. Similarly, we assigned to $C$. 


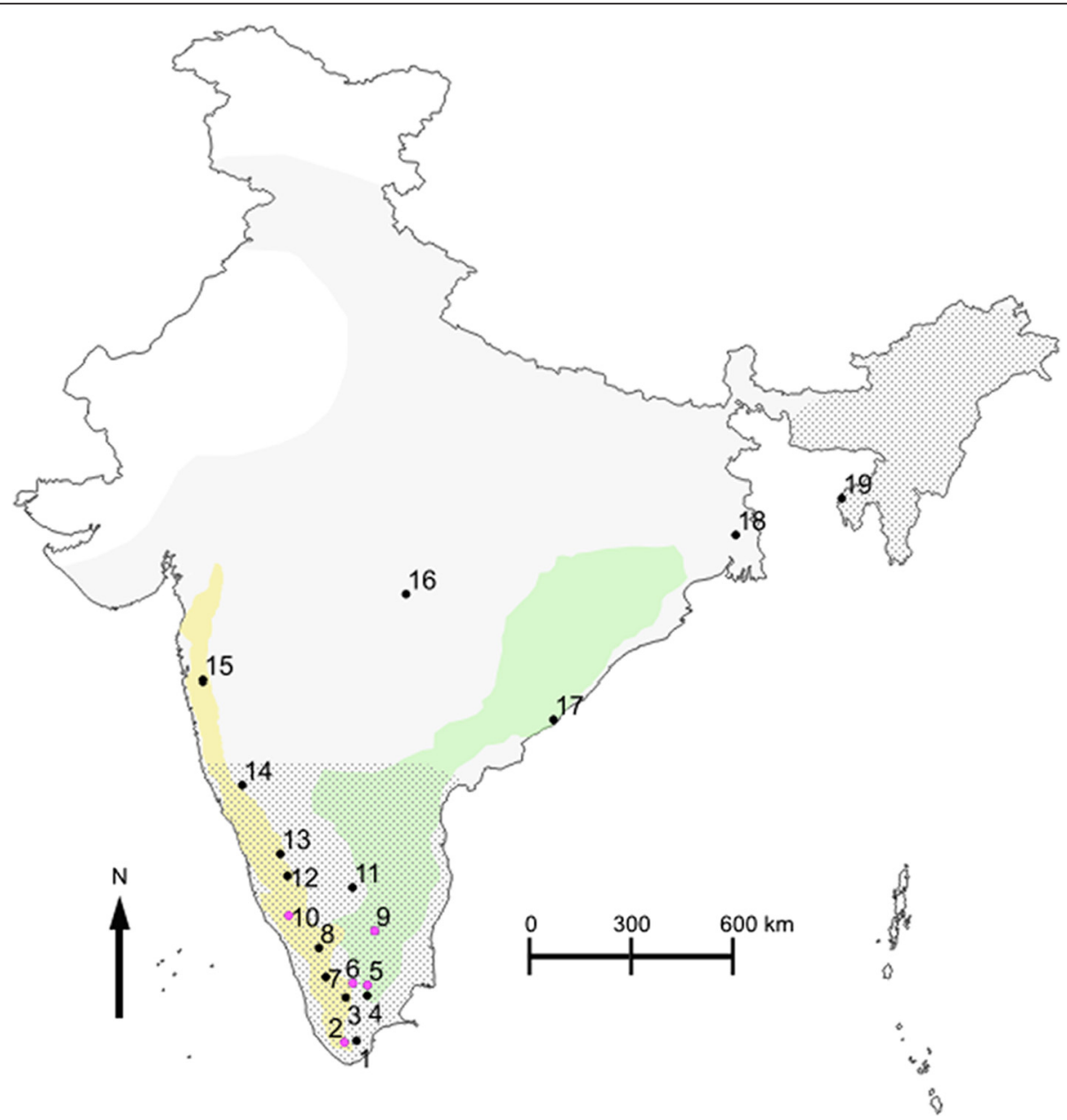

Fig. 1 Map showing the distribution and sampling details of each species. Shaded area corresponds to the range of C. sphinx across India. Dotted areas correspond to the range of C. brachyotis lineage across India. Distribution maps for both species were obtained from International Union for Conservation of Nature. Rajasri Ray provided the shape files of the political boundary of India as well as the boundaries of the Eastern Ghats and Western Ghats. While C. sphinx is found across various habitat types at lower altitudes, C. brachyotis is restricted to mountain ranges [18]. In southern India, C. brachyotis is reported from the Eastern Ghats and the Western Ghats mountain ranges ([18] and the present study). In the map, we have denoted the Eastern Ghats in green and the Western Ghats in yellow. Sampling locations are presented as black dots with numbers corresponding to a population. Locations corresponding to areas of coexistence are marked in pink dots. Details of sampling are provided in Additional file 1: Table S1

$\operatorname{sphin} x$ all bats with a forearm length $\geq 63.4 \mathrm{~mm}$. Individuals that could not be identified as either species were labeled 'unassigned'. We described an area as a contact zone or zone of coexistence when morphologically typical adult individuals of both species were captured either in the same mist net or during multiple sampling sessions in the same location. We used published records [18] alongside our sampling observations to identify locations as allopatric.

We obtained tissue biopsies using a $6 \mathrm{~mm}$ or $4 \mathrm{~mm}$ sterile punch from both wing patagia of an individual and stored them in $95 \%$ ethanol $\left(-20{ }^{\circ} \mathrm{C}\right)$ prior to DNA extraction. All bats captured in mist nets were released immediately after sampling. Bats captured in day roosts were treated following Garg et al. [23]. All sampling protocols were in accordance with the ethical standards of the institutions involved in this research.

\section{DNA extraction and microsatellite genotyping}

We extracted total genomic DNA using a modified saltchloroform extraction protocol following Chattopadhyay et al. [22]. We amplified three tri- and six tetranucleotide repeat loci, previously developed for $C$. sphinx [25], either using Ampli-Taq Gold DNA polymerase (Applied Biosystems, $\mathrm{n}=266$ ) following Chattopadhyay et al. [22] or PCR Master mix (MM, Qiagen, $\mathrm{n}=121$ ) (Additional file 2). We genotyped all samples using the ABI3100 XL platform and scored allele sizes using Genemapper v 4.0 (Applied Biosystems). We normalized post genotyping allele sizes using TANDEM [26], 
which uses a power function to transform allele sizes to integers, while minimizing the rounding errors. Details of error rates and missing data calculations are provided in the supplementary information. Percentage of missing data, number of alleles and allele size range are summarized in Additional file 3: Table S2. We used the normalized allele sizes for subsequent analyses.

\section{SNP generation from ddRAD libraries}

In addition to genotyping microsatellites, we also mined SNPs from genome-wide data using the ddRAD approach [21] (details in Additional file 2). We chose a subset of samples from both species as well as putative introgressed individuals following trends obtained from the microsatellite data (Additional file 1: Table S1). We digested these samples with NlaIII and MluCI enzymes. We used $130 \mathrm{ng}$ to $200 \mathrm{ng}$ of DNA as starting material. Details of library preparation are provided in the Additional file 2. A paired end run in one lane of an Illumina Hiseq1000 was performed with 10pM product from each library. Quality scores (QC) (FastQC, [27]) suggested poor quality in the restriction sites due to low diversity in both the forward and paired end run (Additional file 4: Figure S1). We analyzed only the forward run as we wanted to mine unlinked markers and obtain only a single SNP per locus. We used the STACKS 1.09 [28] pipeline for demultiplexing as well as to obtain SNPs (process_radtag .pl, denovomap.pl and populations.pl). We allowed for one error in the barcode during demultiplexing and trimmed the dataset to $80 \mathrm{bp}$ length. We used the denovomap.pl program in STACKS to call SNPs. The minimum number of reads to call a stack (stack depth) was set at $10(-\mathrm{m})$. Unusually high numbers of identical reads signify repeat regions or regions of gene duplications. In order to remove these regions we allowed breakup of highly repetitive stacks $(-t)$. We allowed for 2 mismatches between the reads within a stack $(-\mathrm{M})$ of an individual and further allowed two mismatches between stacks when comparing across individuals. SNP calling was based on default parameters. We used the population.pl script to generate unlinked SNPs with varying degrees of missing data across individuals. We included a SNP when the locus containing it was present in at least one species, ensuring that both species contribute towards marker generation. This would reduce the effect of ascertainment bias arising from restriction site polymorphism (enhancing intraspecific variation), and account for reduced variability (SNPs are isolated de novo from both species simultaneously).

\section{Mitochondrial sequencing}

We sequenced the entire (1140 bp) cytb gene from a subset of pure individuals of both species as well as unassigned individuals (112 sequences; KF042154-KF042252,
KJ417498-KJ417512). We used a suite of generic primers as well as specific primers designed for this study, to amplify the entire gene (Additional file 2).

\section{Morphological analysis}

We obtained summary statistics of morphological variables. We investigated the morphological variation within our dataset through multivariate analyses of morphological traits. We tested for multivariate normality in $\mathrm{R}$ [29]. We performed multivariate analyses of the morphological variables in the $\mathrm{R}$ package Factomine $\mathrm{R} 1.27$ [30]. We performed PCA using the continuous variables (forearm length, tibia length and ear length). However, we also incorporated the categorical variable ear margin as a supplementary variable to improve clustering [30]. We only considered individuals without missing data for this analysis.

\section{Microsatellite analysis}

We obtained allele numbers and allele size ranges, performed a test for deviation from Hardy Weinberg equilibrium (HWE) (Genepop 4.2.1, [31]) and tested for Linkage Disequilibrium in FSTAT 2.9.3.2 [32] (Additional file 2 ). We checked for the presence of null alleles in the dataset using Microchecker [33], and tested for the presence of homoplasy and ascertainment bias within our dataset (Additional file 2). We performed a test for neutrality in BayeScan 2.1 [34] with default settings. The algorithm divides $F_{S T}$ into a population specific (beta) and a locus specific component (alpha). It looks for significant deviation of the locus specific component from the population specific component. A significant alpha value would suggest that the locus is under selection. We set a prior odd of 10 assuming that the neutral model is 10 times more likely than the selection model at a locus. We used a $5 \%$ cutoff value for the false discovery rate to identify outlier loci. We performed all analyses using genetically pure individuals obtained from an initial assignment test as mentioned below.

We used a model-based clustering approach implemented in STRUCTURE 2.3.4 [35] to identify genetic clusters $(\mathrm{K})$ within our dataset and to quantify the extent of admixture based on our microsatellite loci. STRUCTURE runs were implemented without an a priori assumption of group membership. We ran STRUCTURE from $\mathrm{K} 1$ to 6 with 10 iterations per K. For each iteration we implemented a burnin of 500,000 generations and MCMC for 1,000,000 generations. We first used the second order rate of change of the log probabilities of the data (delta K, [36]) to statistically identify the most likely number of genotypic clusters $(\mathrm{K})$ within the entire dataset. Further, for each $\mathrm{K}$ that we obtained, we evaluated individual ancestry coefficients (q values) to assign individuals into population clusters using CLUMMP 1.1.2 [37]. We 
performed a full search for $\mathrm{K}=2$ and 3 , and used the greedy algorithm (10,000 iterations) for higher $\mathrm{K}$ values.

To assess the capability of our microsatellite loci to distinguish between species and to obtain cutoff values for ancestry coefficients (q values) for pure and admixed individuals, we generated a simulated dataset in Hybridlab [38] and followed Burgarella et al. [39] to obtain estimates of efficiency, accuracy, and type I errors in assigning purebreds and hybrids using STRUCTURE (Additional file 2).

\section{Genome-wide SNP analysis}

We calculated average heterozygosity in Cervus 3.0 [40] and extent of missing data in PLINK [41]. We also tested for deviation from neutrality in BayeScan 2.1 as mentioned earlier and subsequently performed individual assignments in STRUCTURE using only neutral loci (using same conditions as microsatellite analysis). We ran STRUCTURE from $K=1$ to 6 with 10 iterations per K. Every iteration included a burnin of 50,000 generations and MCMC for 100,000 generations. We obtained the optimal K using Evanno's method [36] similar to the microsatellite data.

To test the effect of missing data and number of loci, we obtained SNPs from STACKS with different levels of missing data and assessed trends observed for each dataset. We mined loci if they were present at least in one species; thus the extent of missing data allowed per locus reflects the species level missing data and not the level of missing data in the entire dataset. In this way, we obtained four datasets with the following levels of missing data allowed: $10 \%, 30 \%, 50 \%$ and $70 \%$.

\section{Mitochondrial DNA-based phylogenetic reconstruction}

We aligned our 112 full length cytb sequences with previously published Cynopterus sequences $(\mathrm{n}=12 ; C$. sphinx: FJ489964, FJ489958, JX283292, DQ445703, FJ489972; C. brachyotis Sunda lineage: GU724956; C. brachyotis Phillipines lineage: AB046320, AB046321; C. brachyotis Forest lineage: GQ410210 and C. horsfieldii: EF201637, EF201639 and EF201643) and outgroup taxa ( $\mathrm{n}=7$; Ptenochirus jagori: FJ218480 and GQ410211; Pteropus vampyrus: EF584230 and JN398212; Rhinolophus ferrumequinum: EU436673; Hipposideros bicolor: DQ054808 and Megaderma lyra: DQ888678; alignment length of 996 bp, Additional file 2) and reconstructed the phylogeny of the Cynopterus species complex using a Bayesian paradigm implemented in MrBayes 3.2.5 [42]. We performed two runs with four chains. The swapping frequency and temperature were kept at default values. Trees were sampled every $500^{\text {th }}$ generation and diagnostics were calculated every $5,000^{\text {th }}$ generation for a total of $10,000,000$ generations per run. At this point the standard deviation of the split frequency had reached below 0.01 . We used Tracer v1.5 [43] to check for convergence. We obtained consensus trees from MrBayes using a $50 \%$ burnin and the resultant phylogram was viewed in Figtree 1.4 [44]. Some of the published sequences were very short (690 bp), specifically the Myanmar lineage of C. brachyotis (AY628945), which is represented by only one sequence [3]. To accommodate these sequences and obtain a wider coverage of cynopterine lineages we added these sequences to our dataset and reconstructed a second phylogeny (based on a 690 bp alignment; other GenBank accession id's included: C. sphinx Myanmar lineage: AY629000; C. brachyotis Sunda lineage: AY628945; C. brachyotis Sulawesi lineage: AY628937 and AY628938; C. brachyotis Forest lineage: AY628966) in MrBayes using the same conditions. This second analysis was run for $5,000,000$ generations.

We computed mean between-group and within-group genetic distance in MEGA 5.0 [45]. We also tested for homoplasy (saturation of phylogenetic information) within our dataset using DAMBE [46] (Additional file 2). We did not observe any significant saturation within our dataset (Additional file 3: Table S3).

\section{Genome-wide locus-based phylogenetic analysis}

We also performed a phylogenomic reconstruction with the genome-wide ddRAD dataset. We first isolated concatenated sequence data from the 46 individuals using the pipeline pyRAD [47]. We used demultiplexed raw reads obtained from STACKS (process_radtags.pl) as an input to pyRAD. Thus, data obtained from this pipeline was independent from the data obtained from the STACKS pipeline. We considered the minimum depth at each locus for an individual as 10, restricted the number of undetermined bases allowed per locus to 4 and set the similarity threshold for global and within sample clustering at 0.88 .

We generated four distinct datasets allowing for $10 \%$, $30 \%, 50 \%$ and $70 \%$ missing data (sequence length for each dataset: 770 bp; 9,064 bp; 110,642 bp; and 700,088 bp, respectively). Unlike for the SNP dataset, the missing data cutoff here reflects the actual missing data allowed per locus across all 46 individuals.

We followed a supermatrix approach with concatenated sequence data to reconstruct the phylogeny of our sampled species using maximum likelihood as implemented in RAxML v8 [48]. We used the GTR + gamma model of sequence evolution and performed a single full maximum likelihood tree search, applying the rapid bootstrap algorithm with 1000 replicates to each dataset. The final unrooted tree was viewed in Figtree with midpoint rooting.

\section{Test for introgression}

We obtained Patterson's D statistic to test if a pattern of shared variation between groups can be better explained 
by gene flow than incomplete lineage sorting [49]. This test has been specifically useful in identifying incidents of introgression using SNP based genome-wide datasets [13]. We performed the four taxa D test in the evobiR package in $\mathrm{R}$. This test assumes that the data consist of four clades: two sister clades, one putative admixed clade formed due to possible gene flow between the sister clades and an outgroup clade. It then assesses the shared variation across all SNPs (which are homozygous in the outgroup) that follow an ABBA or BABA pattern between these clades [13, 49-51]. We performed 1000 bootstraps to calculate the standard deviation of the Dstatistic and calculated Z-scores to determine significant introgression ( $\mathrm{Z}$-score $>2.55$ and $\mathrm{p}$ value of two tailed test $<0.01)$. We performed the test for introgression for all four levels of missing data generated in pyRAD.

\section{Discriminant analysis and classification function}

In order to understand if genetic classifications can improve morphological identification of both species, we performed a forward stepwise discriminant analysis (DA, STATISTICA version 10) using genetic data as the dependent variable and the three continuous variables (forearm length, ear length and tibia length) as independent predictors. We performed classification of cases and obtained classification functions.

\section{Results}

Field sampling and morphological analysis

We captured and sampled 405 bats (395 adults: C. sphinx - 230, C. brachyotis - 138 and unassigned - 27) across their ranges in India (Fig. 1, Additional file 1: Table S1). Initial field identifications revealed the presence of both morphologically typical as well as unassigned individuals (Additional file 1: Table S1). We identified two distinct contact zones, one in the Eastern Ghats mountain range (Yercaud, location: 9, Fig. 1) and the other in the southern Western Ghats mountain range (KMTR, Kalakad Mundanthurai Tiger Reserve, location: 2, Fig. 1). In these areas, we often captured both species in the same mist net suggesting an overlap in foraging habitats. We removed juveniles from all morphological analyses (Additional file 1: Table S1).

Morphologically 'unassigned' individuals were mostly captured throughout the higher elevation distributional range of C. brachyotis (average elevation $=1137.6 \mathrm{~m}$, Additional file 1: Table S1). We observed considerable overlap in morphological variables between the two species (Fig. 2 a, b and c, Additional file 3: Table S4). We did not observe sexual dimorphism in these species (Additional file 3: Table S5). The morphological variables were not normally distributed (Jarque-Bera multivariate normality test, $P<0.001)$. PCA analysis based on data from 228 individuals for which we had no missing data

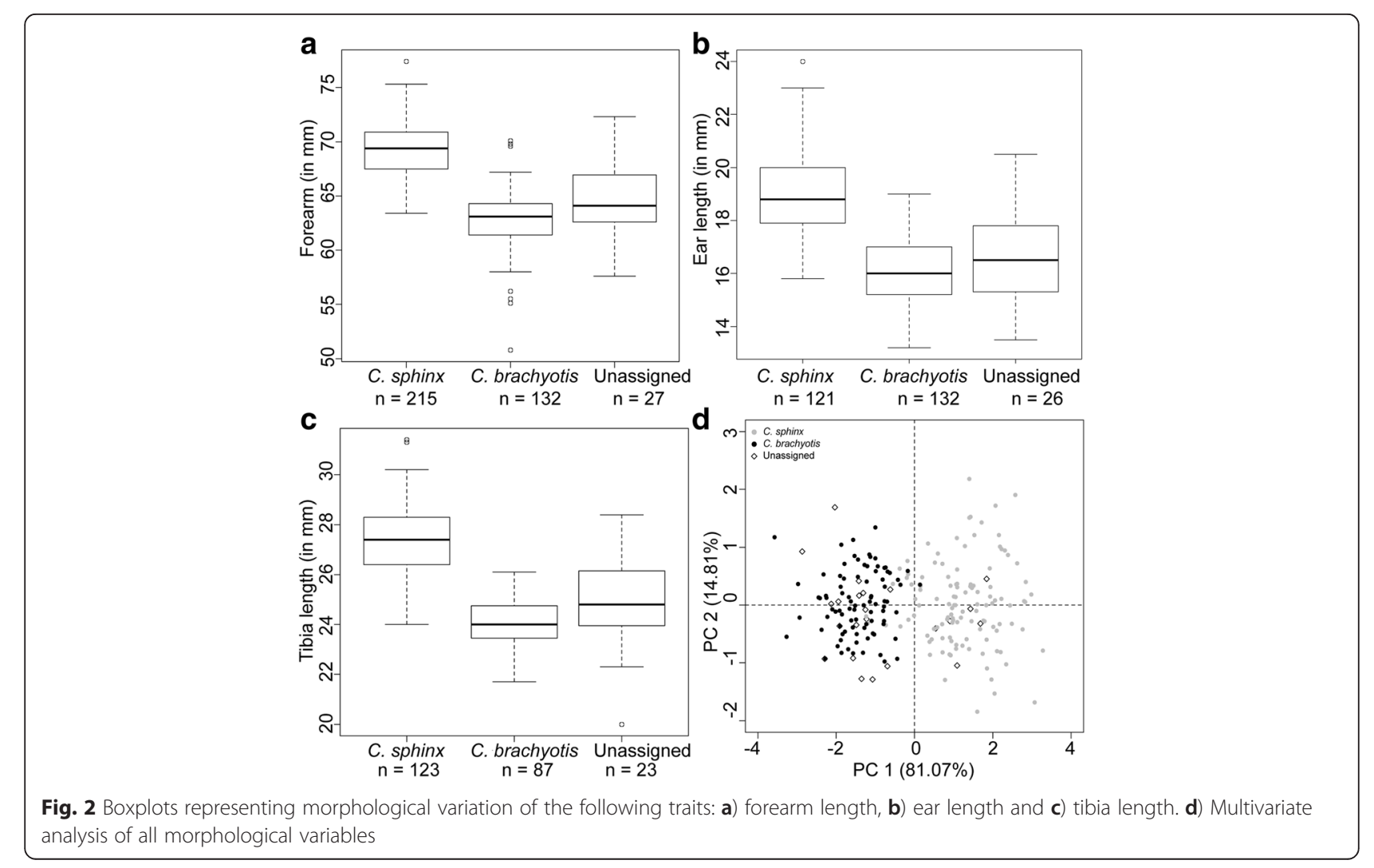


resulted in the first two axes (which explained $96 \%$ of variation; Fig. 2d) differentiating samples into two major clusters with overlap: C. sphinx and C. brachyotis (Fig. 2d). Among the continuous variables, forearm length was the best predictor in the first axis and was strongly correlated with tibia length, followed by ear length (Additional file 5 : Figure S2). Ear length contributed most in the second axis (Additional file 3: Table S6).

\section{Microsatellite analysis}

We genotyped 387 individuals, and overall, our dataset had $0.4 \%$ missing data (Additional file 3: Table S2) affecting eight C. sphinx and four C. brachyotis individuals. Twelve populations out of 19 showed a significant heterozygote deficit, though there was no significant linkage disequilibrium within our dataset. We observed null alleles at various loci within our samples (Additional file 6: Table S7), especially in CSP8 (10 out of 19 populations). We observed homoplasy in our microsatellite dataset, but no ascertainment bias (Additional file 2). We also observed that four loci (CSP2, CSP7, CSP8 and CSP9, Additional file 3: Table S8) did not fit neutral expectations. We therefore performed microsatellite-based analyses both with and without these loci.

STRUCTURE runs with all nine loci revealed that both species neatly segregate into two clusters and that, statistically, $\mathrm{K}=2$ best explains the data (Fig. 3a, b and Additional file 7: Figure S3). One cluster included most of the field identified $C$. sphinx and the other $C$. brachyotis. We further evaluated the average ancestry coefficients (q) of $\mathrm{K}=2$. We used cutoff values for pure individuals of $>0.85$ and $<0.15$ and for intermediates of $\leq 0.85$ and $\geq 0.15$ based on the values obtained from the simulated data (Additional file 2). We observed genetically admixed individuals mainly within allopatric populations $(n=7)$ of both species (Additional file 3: Table S9). Conversely, we observed only one admixed individual in a contact zone (Fig. 3b). None of the genetically admixed individuals had any missing data. We observed that all field based unassigned individuals could be genetically assigned to either of the two species (Fig. 3a, Additional file 3: Table S10A).

STRUCTURE runs using only neutral loci (cutoff values for pure individuals: $>0.70$ and $<0.30$, and for intermediates: $\leq 0.70$ and $\geq 0.30$, Additional file 2) also revealed the presence of 8 genetically admixed individuals. However, there was broad disagreement in assigning admixed individuals between both datasets. Analyses with the five neutral loci revealed additional admixed individuals in the Tirunelveli population which were considered pure in analysis with all loci. Conversely, some individuals which were considered admixed with all loci emerged as
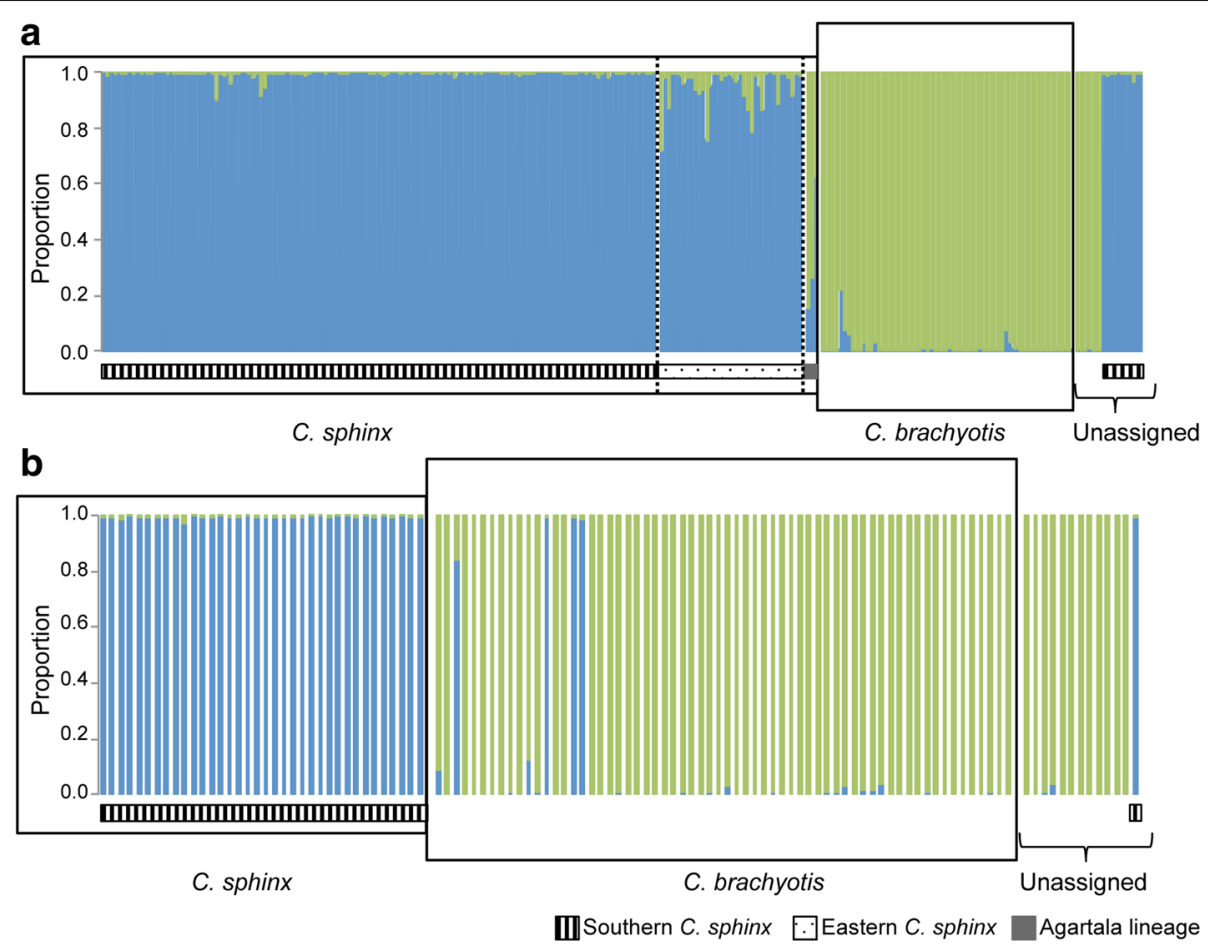

Fig. 3 Barplots of the ancestry coefficient q at $K=2$ (all 9 loci) of individuals belonging to a) populations in allopatry and b) populations in contact zones using microsatellite markers (assignment test with all 9 loci). The vertical axis represents the proportion of the ancestry coefficient of each individual belonging to each species. Intraspecific subdivisions within C. sphinx and the Agartala lineage that were identified from the analyses of SNP data (see text for details) are highlighted 
pure when including only neutral loci (Additional file 3: Table S9).

Microsatellite analysis also revealed the presence of additional contact zones in the hill ranges of the Western and Eastern Ghats (locations: 5, 6 and 10, Fig. 1 and Additional file 3: Table S10A). Genetic assignment test revealed the presence of $C$. sphinx in high altitude regions of both the Western Ghats and the Eastern Ghats (average elevation $=1168.67 \mathrm{~m}$ ). Significantly, we observed that almost the entire sampled distributional range of $C$. brachyotis is part of the contact zone.

Comparisons across various $\mathrm{K}$ values revealed two admixed genomic clusters within $C$. $\operatorname{sphin} x$ at $\mathrm{K}=3$ and $\mathrm{K}=4$, one cluster representing samples from Eastern India (eastern C. sphinx, locations: 17, 18 and 19 in Fig. 1 and Additional file 1: Table S1) and the other cluster representing samples from peninsular and southern India (southern C. sphinx, locations: $1,2,4,5,6,9,10-$ 16 in Fig. 1, Additional file 1: Table S1) (Additional file 8: Figure S4A). Further increase in $\mathrm{K}$ did not reveal any additional biologically clusters (data not shown). When the STRUCTURE analysis was repeated with only neutral loci, we did not observe any biologically relevant sub-structuring (Additional file 8: Figure S4B) possibly suggesting the lack of power of these five loci to identify intraspecific variation.

\section{Genome-wide SNPs}

We obtained 274 million paired-end reads from 46 individuals. In our selection for the ddRADseq data subset, we chose individuals representative of the microsatellite diversity within all species-level lineages based on results from microsatellite STURCTURE analysis. We analyzed only the forward reads (140 million reads), out of which 84 million reads passed the quality filtering criteria (process_radtags). The number of reads per individual ranged from 0.8 million reads to 3.1 million reads, with an average of 1.8 million reads. We obtained 70, 365, 2381, and 10,866 SNPs allowing for $10 \%, 30 \%, 50 \%$ and $70 \%$ missing data, respectively. We detected signs of selection in three loci from the $30 \%$ missing data dataset and in 20 loci from the $50 \%$ missing data dataset, but none in the other two datasets. The average heterozygosities and levels of missing data per locus and per individual are summarized in the Additional file 3: Table S11.

We used q value cutoffs based on previous trends obtained from the microsatellite dataset to denote pure and admixed categories in the assignment tests. We considered individuals with $\mathrm{q}$ values $>0.85$ and $<0.15$ as pure and individuals with $\mathrm{q}$ values from 0.15 to 0.85 as admixed. The most probable number of clusters was found to be 2 . We compared trends across each $\mathrm{K}$ value for all four datasets and also compared between the Ks' of each dataset. The results reveal that at $\mathrm{K}=2$, for all levels of missing data, the SNPs could distinguish between the species as well as retrieve intraspecific variation in agreement with microsatellite data. However, eastern Indian $C$. $\operatorname{sphin} x$ group (eastern C. $\operatorname{sphin} x$, locations: 17,18 and 19 in Fig. 1 and Additional file 1: Table S1) emerged with variable affinities and levels of purity (Fig. 4), as a distinct cluster from samples from southern and peninsular India C. sphinx group (southern C. sphinx, locations: 1, 2, 4, 6, 9, 10-15 in Fig. 1, Additional file 1: Table S1). Specifically, in the eastern C. sphinx group the genomic contribution of the C. sphinx cluster increased with a rise of missing data and the concomitant increase in the number of loci. In comparison, assignments with $\mathrm{K}=3$ apportioned the third genetic cluster to a homogeneous eastern C. sphinx group in most datasets (except for the analysis with the least number of loci; Fig. 4b). $K=4$ revealed further substructuring in dividing out two individuals from Agartala (Agartala cluster, location: 19, Additional file 1: Table S1) as distinct, with increasing levels of purity as the number of loci increases (Fig. 4c). A third individual from Agartala emerged with possible admixed ancestry between the eastern C. sphinx cluster and the Agartala cluster. It has to be noted that allowing for more missing data in STACKS does not result in a significant increase in missing data (Additional file 3: Table S11), but rather in a pronounced increase in the number of loci available for analysis. Similar to the microsatellite dataset, we did not observe any biologically relevant clusters for $\mathrm{K} 5$ and $\mathrm{K} 6$ (data not shown).

We also used STRUCTURE to obtain net nucleotide distances between the four clusters at $K=4$ for the $50 \%$ missing dataset. We took an average of all ten iterations and observed that the Agartala cluster is almost equidistant from the eastern C. sphinx cluster and the C. brachyotis cluster and most distant from southern $C$. $\operatorname{sphinx}$ (Additional file 3: Table S12). Both clusters of $C$. $\operatorname{sphin} x$ were genetically very similar compared to the other clusters.

\section{Mitochondrial DNA-based phylogenetic reconstruction}

In agreement with Campbell et al. [3], our cytb tree supported monophyly of the Indian $C$. brachyotis lineage as well as the C. sphinx lineage (Fig. 5 a and b). We also observed a general agreement between clade membership in the cytb phylogeny, and clustering of microsatellites and SNPs in assignment tests. For all the putative admixed individuals, cyt $b$ clade membership was identical to field identifications (Additional file 3: Table S10B). Individuals that remained unassigned in the field (Fig. 5a) were grouped in clades in agreement with the microsatellite and SNP data (Additional file 3: Table S10). We observed that the Indian C. brachyotis lineage is basal to all other sampled cynopterines (in agreement with Campbell et al. [3]). Genetic distance analysis $(\operatorname{TrN}+\mathrm{G}$ substitution 


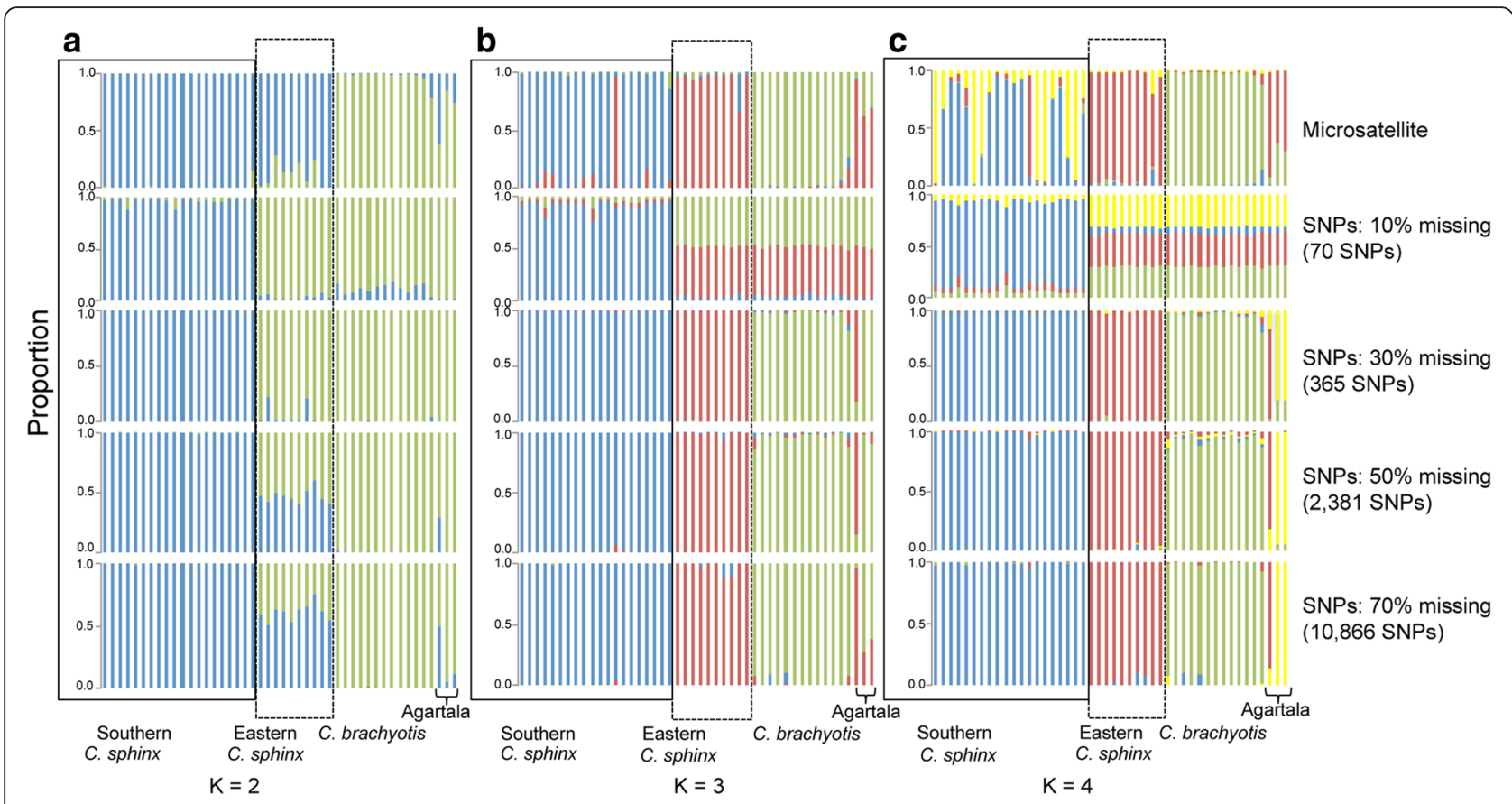

Fig. 4 Barplots of the ancestry coefficient q of microsatellite (assignment with all 9 loci) and various SNP datasets at $\mathbf{a}) K=2, \mathbf{b}) K=3$ and $\mathbf{c}$ ) $K=4$. Intraspecific subdivisions within C. sphinx (eastern C. sphinx and southern C. sphinx) and the Agartala lineage were identified from the comparative analysis of SNP dataset and are specified accordingly
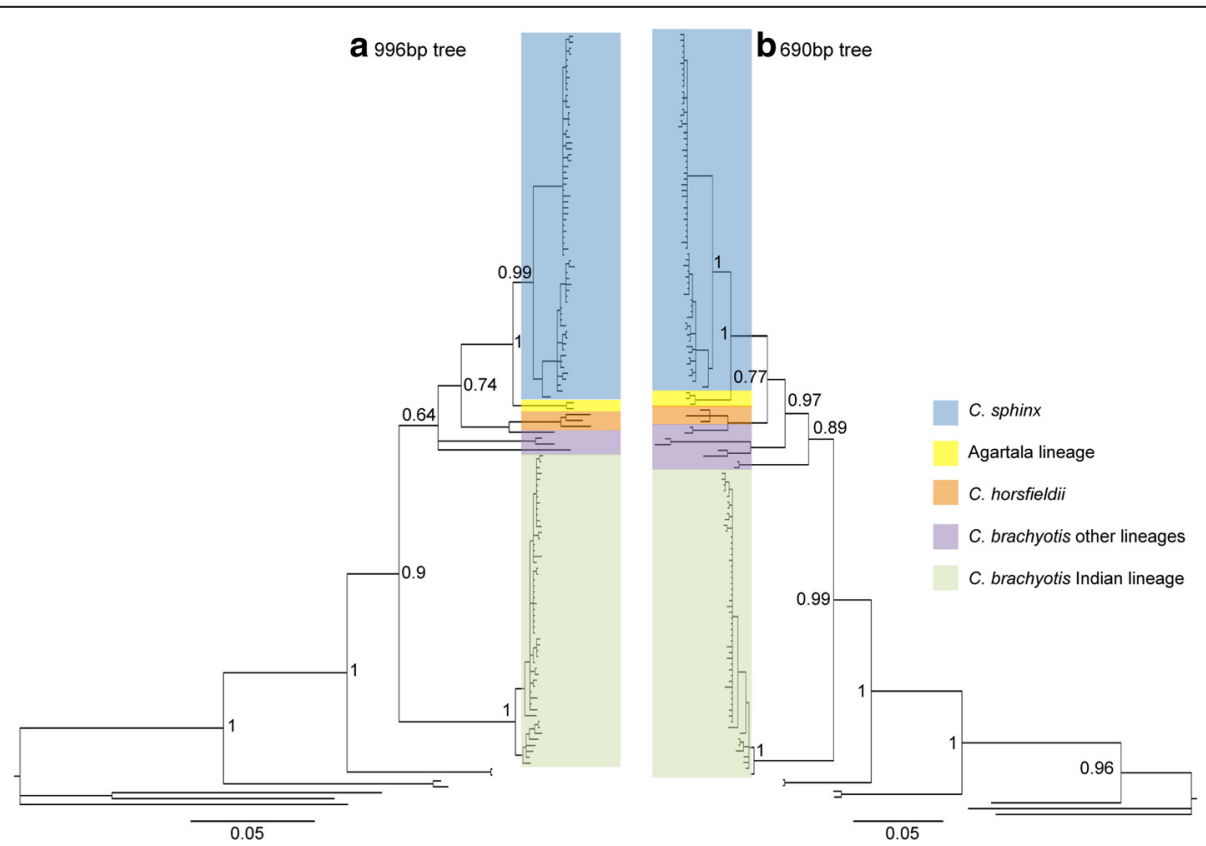

Fig. 5 Phylogenetic tree of cytb sequences a) 996 bp from 131 samples; b) 690 bp from 136 samples. The node labels represent posterior probability support for the respective node. The clade colored blue represents C. sphinx, yellow represents the Agartala lineage (in Fig. 5b, it also consists of a single sequence representing the C. brachyotis Myanmar lineage), orange represents C. horsfieldii, purple color represents all the other lineages of C. brachyotis and green represents C. brachyotis from India 
model) showed as much as a $9.4 \%(0.094)$ divergence between the two species in agreement with Campbell et al. [3] (Additional file 9: Table S13). However, two individuals from the Agartala cluster (as based on SNP data) formed a separate monophyletic clade with high genetic divergence from the other C. sphinx lineages (0.04). Their average distance from the Indian C. brachyotis lineage (0.11) was also higher than that between the $C$. sphinx clade and the Indian $C$. brachyotis lineage (0.094). In the partial cytb (690 bp) tree, which incorporated additional extra-limital samples, these individuals formed a separate clade with $C$. brachyotis from Myanmar, whereas C. sphinx from Myanmar was part of the C. sphinx clade (Fig. 5b). The putative admixed individual formed part of the C. sphinx clade and had a genetic distance of 0.019 with other $C$. sphinx and 0.052 with the Agartala cluster.

\section{Phylogenetic reconstruction from genome-wide data}

Phylogenetic reconstructions of the concatenated genome-wide sequence data with different levels of missing data unambiguously separated the species as distinct clades. Clade identities were identical to those observed in the cyt $b$ phylogeny and genetic assignments with SNPs and microsatellites. Both species segregated as monophyletic clades when we allowed for $10 \%$ missing data (Fig. 6). When we allowed more loci and sampled more regions of the genome, we observed that the two individuals representing Agartala cluster formed a monophyletic clade basal to all $C$. sphinx sequences in agreement with the cytb tree (Fig. 6). Though the partial cyt $b$ tree (which contained Myanmar material) classified Agartala individuals as part of the Myanmar C. brachyotis lineage, yet the absence of genomic data from the Myanmar C. brachyotis lineage restricts us from validating the congruence between nuclear genome phylogeny and mitochondrial genome phylogeny. Hence we categorize the Agartala cluster as the Agartala lineage. Further, with $50 \%$ and $70 \%$ missing data and more loci incorporated, the $C$. sphinx clade again subdivides into two reciprocally monophyletic clades, one representing the southern $C$.

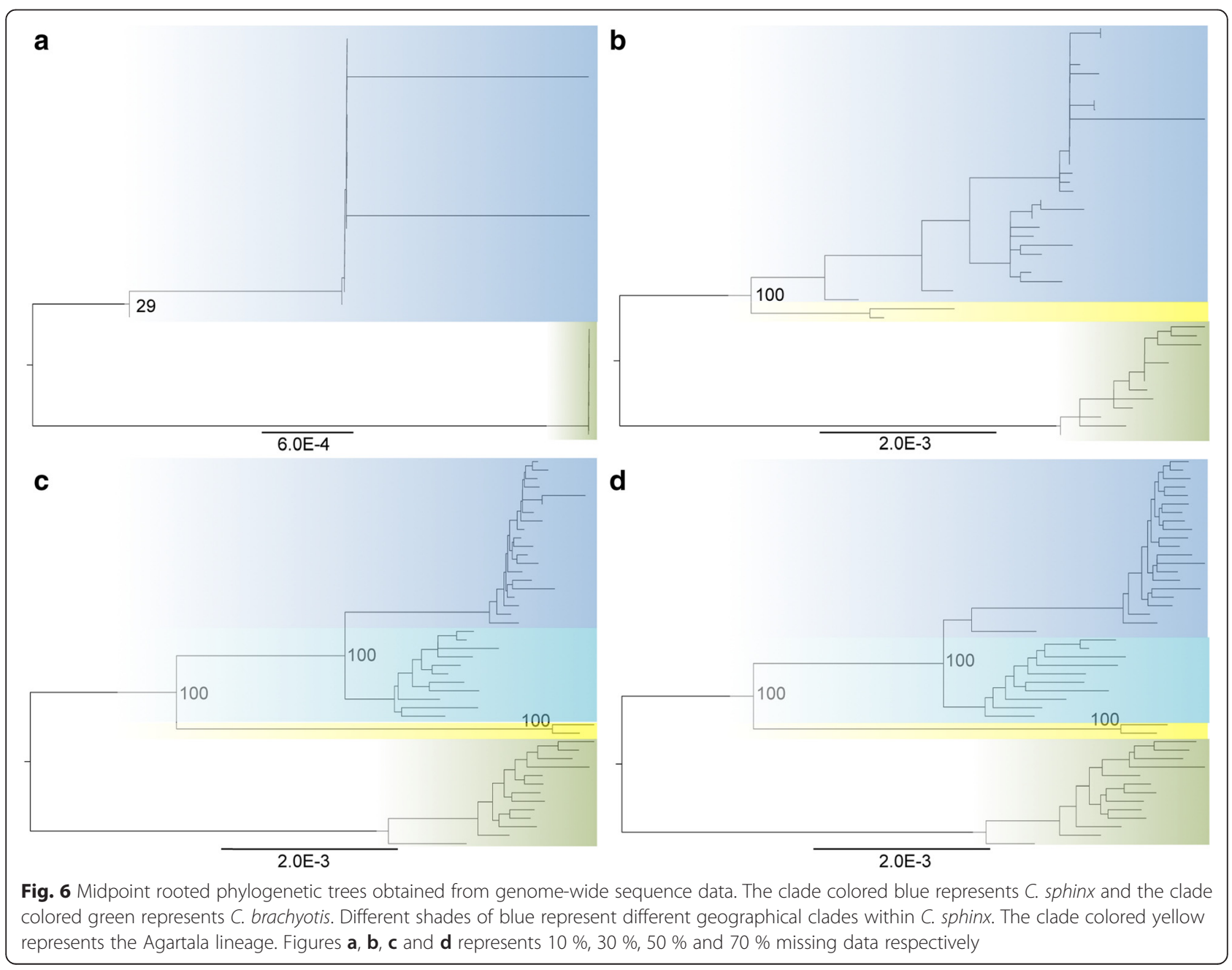


$\operatorname{sphin} x$ and the other representing the eastern C. sphinx in agreement with the genomic clusters (SNP data) identified using STRUCTURE analysis, but in contrast to the cyt $b$ phylogeny, which lacked such resolution.

\section{Test for introgression}

Using the $30 \%, 50 \%$ and $70 \%$ missing datasets (the $10 \%$ missing dataset did not contain any ABBA-BABA site), we performed tests of introgression for two different scenarios (Fig. 7) to investigate gene flow between $C$. sphinx and the Agartala lineage. We used Indian $C$. brachyotis as an outgroup. We first tested for possible introgression in one individual from Agartala (CA008), which was genomically a member of the eastern C. sphinx group with admixture from the Agartala lineage (represented by the other two Agartala individuals) (Figs. 4 and 7a). A significant difference between the number of ABBA and BABA sites suggested introgression (Table 1). For the second test we removed this introgressed individual and performed a test of gene flow between the Agartala lineage and the eastern C. sphinx cluster (Fig. 7b). We obtained similar results of significant differences between ABBA and $\mathrm{BABA}$ sites suggesting gene flow between these two lineages (Table 1).

\section{Genetic data improves morphological classification}

We used the cyt $b$ clade identity as a grouping variable $(\mathrm{n}=112)$. We performed a forward stepwise DA considering $\mathrm{F}$ to enter as $0.010, \mathrm{~F}$ to remove as 0.0 and the minimum tolerance as the default 0.01 . We grouped our data into three categories, C. sphinx, C. brachyotis and the Agartala lineage. The best-fit model consisted of all three morphological variables with forearm length being the best explanatory variable (Additional file 3: Table S14). We further performed self-classification considering an equal probability of group membership. Overall $81 \%$ of the samples $(66.67 \%$ of C. sphinx and $92.5 \%$ of C. brachyotis) could be correctly identified based on morphology. The discriminant function had $100 \%$ power to classify the two individuals from the Agartala lineage.

\section{Discussion}

We investigated morphological and genetic differentiation of congeneric fruit bats and assessed the concordance between these two types of data. Our results reveal the importance of molecular markers, specifically genome-wide markers, in the discovery of cryptic diversity, leading to improved species identification and the documentation of significant range extensions. More importantly, we uncover an additional, hitherto unrecognized, cryptic lineage of Cynopterus coexisting with C. sphinx in northeastern India, and we identify individuals that bear the hallmark of introgression (based on genome-wide DNA) between these two lineages. These results provide the first detailed insights into the complicated patterns of differentiation

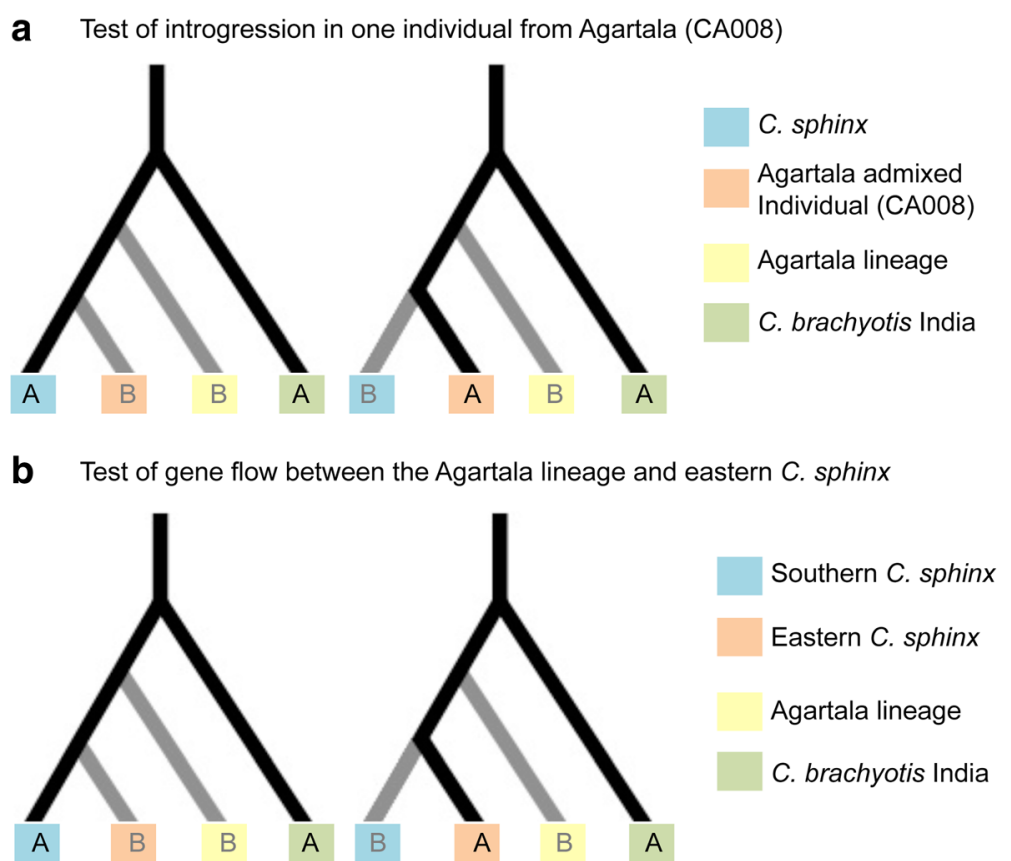

Fig. 7 Graphical representation of clade arrangements for tests of introgression using Patterson's D statistic: a) test of introgression in one individual from Agartala (CA008) and $\mathbf{b}$ ) test of gene flow between the Agartala lineage and eastern C. sphinx 
Table 1 Summary of Patterson' D test statistic. Significant values of D statistic are indicated in bold font

\begin{tabular}{|c|c|c|c|c|}
\hline \multirow{2}{*}{$\begin{array}{l}\text { Dataset from } \\
\text { pyRAD pipeline }\end{array}$} & \multicolumn{2}{|c|}{ Introgression in one individual from Agartala (CA008) } & \multicolumn{2}{|c|}{ Gene flow between the Agartala lineage and eastern C. sphinx } \\
\hline & D statistic & Z score & D statistic & Z score \\
\hline $10 \%$ missing & NA & NA & NA & NA \\
\hline $30 \%$ missing & -0.41 & 9.66 & 0.33 & 5.04 \\
\hline $50 \%$ missing & -0.49 & 94.15 & 0.27 & 34.42 \\
\hline $70 \%$ missing & -0.47 & 221.32 & 0.15 & 60.71 \\
\hline
\end{tabular}

among this cryptic bat radiation and significantly expand our knowledge of their biogeography and levels of reproductive isolation.

\section{Morphology-based identification is unreliable in contact zones}

Available literature suggests considerable overlap in morphology between cynopterine species and advocates the use of genetic assignment tests for identification in such situations $[18,19]$. Our study reiterates that a suite of external morphological characters widely used for species level identification may not be very informative, specifically in contact zones (Fig. 2d). The situation is particularly problematic in peninsular and southern India. Clinal morphological variation [52] within $C$. $\operatorname{sphinx}$ might be an important reason for such low classification power. Future sampling of both species across elevational gradients may reveal the extent of morphological similarity. Additionally, more sampling and cross validation is required to understand the difference between $C$. sphinx and the Agartala lineage. We propose that wherever possible cyt $b$ sequencing or more specifically genome-wide SNP data should be generated to obtain species level assignment.

\section{Cryptic diversity of cynopterine bats in India}

Genome-wide SNPs revealed discrete geographic lineages within C. $\operatorname{sphinx}$ in addition to a cryptic, hitherto unrecognized cynopterine lineage in northeastern India which coexists at least with C. $\operatorname{sphinx}$ (Fig. 4). Further, phylogenetic reconstructions reveal that the Agartala lineage is a sister species of $C$. $\operatorname{sphinx}$ and shares close genetic and phylogenetic proximity with the C. brachyotis Myanmar lineage (Fig. 5b). However, our understanding of the taxonomy of the Agartala lineage is limited due to the lack of a voucher specimen as well as due to the lack of comparative nuclear genomic material from C. brachyotis Myanmar. Future studies should address these issues and provide appropriate taxonomic revisions.

\section{Gene flow and introgression between cynopterine bats in India}

Fruit bats of the genus Cynopterus often share large contact zones [3, 4, 20,53]. We identified and assessed contact zones of C. sphinx and C. brachyotis in India and found no strong evidence of hybridization in these zones based on genome-wide SNPs; in contrast microsatellite data were inconclusive, probably based on their considerably lower level of resolution as compared to genome-wide SNPs (Figs. 3 and 4). Interestingly, SNP data revealed instances of gene flow between C. sphinx and the Agartala lineage. A comparison of mtDNA phylogeny, assignment tests and shared variability (ABBA-BABA tests) suggests at least one incidence of male-mediated introgression (CA008) from the Agartala lineage into the nuclear genome of C. sphinx. CA008 is an adult male with $C$. sphinx cytb haplotype and a high nuclear contribution of $C$. sphinx. Additional tests further revealed hallmarks of introgression from the cryptic Agartala lineage into the eastern cluster of C. $\operatorname{sphinx}$, including those individuals whose genome-wide SNP profile had appeared pure (Figs. 4 and $7 \mathrm{~b}$ ). The gene flow between these two lineages may be limited, therefore resulting in a level of introgression that is undetectable with genomic scans spanning thousands of loci and is only detected through specific tests that can distinguish between introgression and incomplete lineage sorting [49].

It is difficult to ascertain the relative extent of contemporary and historical gene flow between $C \cdot \operatorname{sphinx}$ and the Agartala lineage as the test of shared variation lacks sufficient power [51]. The low sample size of the Agartala lineage within our dataset also prevents us from making a coalescent based model comparison to assess the pattern, extent and direction of gene flow between these two species. More extensive sampling in northeastern India and the Indo-Myanmar biodiversity hotspot is required to further unravel the evolutionary affinity of this lineage as well as systematically characterize patterns of gene flow between the two lineages.

Previous evolutionary inquiries into $C$. $\operatorname{sphinx}$ and other southeast Asian lineages of $C$. brachyotis could not obtain conclusive evidence of interspecies gene flow or the lack of it [53]. However in the light of the higher resolution provided by genome-wide data it will be interesting to revisit gene flow between various species of cynopterine fruit bats across their range in the Paleotropics, specifically since this group has experienced a very recent radiation and much of the interspecific relationships are polytomous and unresolved $([3,16]$ and this study), thereby increasing the possibility of ancient gene flow during and/or after divergence. Additionally, the 
distributions of most of cynopterine species are nested within the broad distribution of $C$. sphinx, suggesting that the evolutionary history of C. sphinx may include complicated scenarios of admixture with different lineages. Our results using genome-wide DNA evidence lend support to previous case studies of interspecific hybridization in bats that have revealed gene flow in contact zones [54-57]. The lack of gene flow between $C$. sphinx and C. brachyotis in peninsular and southern India may be an artifact of insufficient sampling, as studies may miss rare admixture. However, lack of genomic data from an appropriate outgroup species in the current study also prevents us from further examination of low levels of admixture. More population and genomic sampling may provide additional insight into patterns of isolation between these two species.

\section{Genome-wide SNPs: more loci provide better resolution}

One important trade-off while using a restriction enzyme based reduced representation library of genome-wide data is between the extent of missing data and the number of loci. Reducing missing data inevitably reduces the number of loci quite drastically, specifically when data from a single lane is analysed. However, recent studies have shown that mining more loci regardless of a significant amount of missing data may still provide more power to the data than sampling fewer loci with less missing data [10, 58]. A comparison of observations across various levels of missing data in our study also reveals that mining more loci with missing data rather than fewer loci that lack missing data can provide singnificantly more biologically relevant information in both population genomic and phylogenomic analyses (Figs. 4 and 6).

\section{Contrasting microsatellite/mitochondrial and genome- wide information}

Although the microsatellite markers used in this study are too few in number and suffer from multiple drawbacks, they are able to identify purebred individuals. However, they suffer from low power and efficiency when identifying admixture (Additional file 3: Table S15). The ddRADseq data fare considerably better and reveal subtle intraspecific sub-structuring. Thus, future assignment studies may consider the generation and analyses of genome-wide loci as performed in this study. This is of particular advantage specifically because a few hundred individuals can be sequenced in a single lane. Mitochondrial cyt $b$ data were also effective in assigning individuals to species level and can be used for initial identification purposes, but they are inherently problematic in the assignment of admixed individuals as they will only reveal information about the matrilineal ancestry.

\section{Conclusion}

Our study uses thousands of genome-wide markers from natural populations of Old World fruit bats to address the complex evolutionary dynamics of a recent radiation. Our SNP data identified an unrecognized, cryptic lineage of cynopterine fruit bat in northeastern India, and provided evidence of admixture and introgression between C. sphinx and this cryptic lineage. Our results suggest caution when using standard external morphological traits in species identification within the cynopterine radiation, especially for the broadly distributed C. $\operatorname{sphinx}$, and emphasizes the utility of genetic markers in species identification when morphology is inaccurate. The use of large number of genetic markers not only improves assignment of individuals at the species level, but also uncovers fine-scale genetic differentiation patterns which maybe particularly important when studying species with large distributional ranges. Using fewer genetic markers (microsatellites as well as SNPs) in such cases may lead to misinterpretation of intraspecific differentiation as gene flow.

\section{Availability of data and material}

Datasets supporting the results of this article are available in GenBank (KF042154-KF042252 and KJ417498KJ417512) and Sequence Read Archive (SRP047152). Microsatellite genotype data is available as Additional file 10.

\section{Additional files}

Additional file 1: Table S1. Sampling details consisting of information about number of individuals typed for each marker. Populations with mutiple sampling points are represented by one representative coordinate. $\mathrm{FA}=$ forearm length, $\mathrm{EL}=$ ear length and $\mathrm{TIB}=$ tibia length All morphological measurements are recorded in millimeters $(\mathrm{mm})$. (XLSX $13 \mathrm{~kb}$ )

Additional file 2: Supplementary methods and results. (DOCX $111 \mathrm{~kb}$ )

Additional file 3: Table S2. Rounding error of each microsatellite locus, the proportion of missing data, number of alleles and allele size range of each microsatellite locus. $b p=$ base pair. Table S3. Test for the saturation of phylogenetic signal in the mitochondrial DNA dataset. Table S4. Summary statistics of morphological variables. $\mathrm{N}=$ Number of samples; SD = Standard Deviation. Table S5. Tests to assess the presence of size dimorphism within each species. Table S6. Contribution of morphological variables in each PCA dimension. Table S8. Table summarizing the results of the test for neutrality. Values in bold indicate loci under selection. We used a $5 \%$ false discovery rate (FDR) to determine loci under selection. The $q$ value is an FDR analog of $p$ value. Table S9. Ancestry coefficient ( $q$ value) values of genetically admixed individuals. Table S10. Details showing genetic affinities of A) morphological unidentified individuals in field and B) genetic intermediates identified using all nine loci. Table S11. Average

heterozygosity and average missing data of various SNP datasets obtained from STACKS. Table S12. Net nucleotide distance between different genetic clusters $(K=4)$ of the genome-wide SNP data (50 \% missing dataset). Table S14. Summary statistics obtained from discriminant function analysis [Step 3, number of variables in the model: 3; Wilks' Lambda: 0.3 approx. F $(6,140)$ $=13.97, \mathrm{p}$ value $<0.001]$. Table S15. Power and accuracy of the STRUCTURE program in detecting purebreds and hybrids from simulated microsatellite datasets. SD = standard deviation; HP = hybrid proportion. (DOCX $116 \mathrm{~kb}$ ) 
Additional file 4: Figure S1. FastQC report of the ddRAD run. (PDF $3067 \mathrm{~kb}$ )

Additional file 5: Figure S2. Correlation circle of the continuous variables. (PDF $536 \mathrm{~kb}$ )

Additional file 6: Table S7. Population wise assessments of null alleles and deviation from Hardy-Weinberg equilibrium. (XLS $26 \mathrm{~kb}$ )

Additional file 7: Figure S3. STRUCTURE harvester results. A) Mean estimate of log likelihood ( $(n P(D))$ of each $K, B)$ Estimate of delta K. (PDF $306 \mathrm{~kb}$ )

Additional file 8: Figure S4. Barplot of the ancestry coefficient $\mathrm{q}$ at $\mathrm{K}=2,3$ and 4 of A) all nine loci and B) neutral loci. (PDF $751 \mathrm{~kb}$ )

Additional file 9: Table S13. Net interspecies genetic distances of the cytb dataset. All distances were calculated using the TrN + G model. (XLSX $38 \mathrm{~kb})$

Additional file 10: Microsatellite genotypes used in this study. (XLSX $73 \mathrm{~kb})$

\section{Competing interests}

The authors declare no conflict of interest.

\section{Authors' contributions}

$B C$ and SK conceived the work; BC and SK planned the research design; BC DPS, AKVK and SK took part in field expedition and sample collection; BC KMG and AKVK performed lab work; BC, KMG, FER and UR participated in data analyses and manuscript preparation; DPS, AKVK and SK provided critical inputs in the drafting of the manuscript. All authors approve the final version of the manuscript.

\section{Acknowledgements}

This study was supported by DBT (BT/PR-9382/BCE/08/568/2007) and UGC (1-13/2002 (NS/PE) and F.5-4/2008 (SAP-II)) grants to SK, a DST and NCBS grant to UR and NUS grants to FER ((DBS)WBS R-154-000-583-651, (FS)WBS R-154-000-570-133). BC acknowledges fellowship support from UGC, DST grant to UR and DAE grant to UR. UR thanks the Ramanujan fellowship and the DAE outstanding scientist award for support. BC thanks R Dhanabalan, $S$ Sivaraja, D K Bharti, Kadambari Deshpande, Neelima Kumar, Pooja Murlidharan, Megha Budhwani and Arindam Chatterjee for their support during field works, and N R Venkatesh and Sandeep Rana for helping in lab work. BC and KMG thank Dr. Arjun Shivsundar for his inputs in a previous version of the manuscript, Dr. Rajasri Ray (Centre for Ecological Sciences, Indian Institute of Science) for her help with map preparation, CCAMP for Illumina sequencing (Project ID: BT/ PR3481/INF/22/140/2011) and Goutham Atla for helping with bioinformatics analysis. BC and SK thank Dr. K Anbalagan, Yercaud and Kardana Estate, Megamalai for support during field sampling. The authors thank Fiona Savory, Amruta Varudkar and Meghana Natesh for their comments.

\section{Author details}

${ }^{1}$ School of Biological Sciences, Madurai Kamaraj University, Madurai, India. ${ }^{2}$ Ecology and Evolution, National Centre for Biological Science, TIFR, Bangalore, India. ${ }^{3}$ Department of Biological Sciences, National University of Singapore, Singapore, Republic of Singapore.

Received: 30 July 2015 Accepted: 22 January 2016 Published online: 18 February 2016

\section{References}

1. Bickford D, Lohman DJ, Sodhi NS, Ng PK, Meier R, Winker K, et al. Cryptic species as a window on diversity and conservation. Trends Ecol Evol. 2007;22:148-55

2. Allendorf FW, Hohenlohe PA, Luikart G. Genomics and the future of conservation genetics. Nat Rev Genet. 2010;11:697-709.

3. Campbell P, Schneider CJ, Adnan AM, Zubaid A, Kunz TH. Phylogeny and phylogeography of Old World fruit bats in the Cynopterus brachyotis complex. Mol Phylogenet Evol. 2004;33:764-81.

4. Campbell P, Schneider CJ, Adnan AM, Zubaid A, Kunz TH. Comparative population structure of Cynopterus fruit bats in peninsular Malaysia and southern Thailand. Mol Ecol. 2006;15:29-47.
5. Morningstar CR, Inoue K, Sei M, Lang BK, Berg DJ. Quantifying morphological and genetic variation of sympatric populations to guide conservation of endangered, micro-endemic springsnails. Aquat Conserv Mar Freshw Ecosys. 2014;24:536-45.

6. Stuart BL, Inger RF, Voris HK. High level of cryptic species diversity revealed by sympatric lineages of Southeast Asian forest frogs. Biol Lett. 2006;2:470-4.

7. Lukhtanov VA, Dantchenko AV, Vishnevskaya MS, Saifitdinova AF. Detecting cryptic species in sympatry and allopatry: analysis of hidden diversity in Polyommatus (Agrodiaetus) butterflies (Lepidoptera: Lycaenidae). Biol J Linn Soc. 2015;116:468-85.

8. Manel S, Gaggiotti OE, Waples RS. Assignment methods: matching biological questions with appropriate techniques. Trends Ecol Evol. 2005:20:136-42.

9. Avise JC. Perspective: conservation genetics enters the genomics era. Conserv Genet. 2010;11:665-9.

10. Wagner CE, Keller I, Wittwer S, Selz OM, Mwaiko S, Greuter L, et al. Genome-wide RAD sequence data provide unprecedented resolution of species boundaries and relationships in the Lake Victoria cichlid adaptive radiation. Mol Ecol. 2013:22:787-98.

11. Nadeau NJ, Whibley A, Jones RT, Davey JW, Dasmahapatra KK, Baxter SW, et al. Genomic islands of divergence in hybridizing Heliconius butterflies identified by large-scale targeted sequencing. Philos T Roy Soc B. 2012;367:343-53.

12. Hohenlohe PA, Bassham S, Etter PD, Stiffler N, Johnson EA, Cresko WA Population genomics of parallel adaptation in threespine stickleback using sequenced RAD tags. PLoS Genet. 2010;6(2):e1000862.

13. Rheindt FE, Fujita MK, Wilton PR, Edwards SV. Introgression and phenotypic assimilation in Zimmerius flycatchers (Tyrannidae): population genetic and phylogenetic inferences from genome-wide SNPs. Syst Biol. 2014;63:134-52.

14. Jennings $H$, Wallin K, Brennan J, Del Valle A, Guzman A, Hein D, Hunter S, Lewandowski A, Olson S, Parsons H. Inbreeding, low genetic diversity, and spatial genetic structure in the endemic Hawaiian lobeliads Clermontia fauriei and Cyanea pilosa ssp. longipedunculata. Conserv Genet. 2015; 1-6.

15. Narum SR, Buerkle CA, Davey JW, Miller MR, Hohenlohe PA. Genotyping-bysequencing in ecological and conservation genomics. Mol Ecol. 2013;22:2841-7.

16. Almeida FC, Giannini NP, DeSalle R, Simmons NB. Evolutionary relationships of the old world fruit bats (Chiroptera, Pteropodidae): Another star phylogeny? BMC Evol Biol. 2011;11:281.

17. Campbell P, Schneider CJ, Zubaid A, Adnan AM, Kunz TH. Morphological and ecological correlates of coexistence in Malaysian fruit bats (Chiroptera: Pteropodidae). J Mammal. 2007;88:105-18.

18. Bates PJJ, Harrison DL. Bats of the Indian subcontinent. Sevenoaks, Kent, U.K.: Harrison Zoological Museum; 1997.

19. Bumrungsri S, Racey PA. Field discrimination between lesser short-nosed fruit bat (Cynopterus brachyotis Müller, 1838) and greater short-nosed fruit bat (C. sphinx Vahl, 1797)(Chiroptera: Pterpodidae) in southern Thailand. Nat Hist Bull Siam Soc. 2005;53:111-21.

20. Storz JF, Kunz TH. Cynopterus sphinx. Mamm Species. 1999:613:1-8.

21. Peterson BK, Weber JN, Kay EH, Fisher HS, Hoekstra HE. Double digest RADseq: an inexpensive method for de novo SNP discovery and genotyping in model and non-model species. PLoS One. 2012;7:e37135.

22. Chattopadhyay B, Garg KM, Doss PS, Ramakrishnan U, Kandula S. Molecular genetic perspective of group-living in a polygynous fruit bat, Cynopterus sphinx. Mammal Biol. 2011:76:290-4.

23. Garg KM, Chattopadhyay B, Doss D, A K V, Kandula S, Ramakrishnana U. Promiscuous mating in the harem-roosting fruit bat, Cynopterus sphinx. Mol Ecol. 2012; 21:4093-4105.

24. Brunet-Rossinni A, Wilkinson G. Methods for age estimation and the study of senescence in bats. In: Kunz TH, Parsons S, editors. Ecological and behavioral methods for the study of bats. Baltimore, MD: Johns Hopkins University Press; 2009. p. 315-25.

25. Storz JF. Variation at tri-and tetranucleotide repeat microsatellite loci in the fruit bat genus Cynopterus (Chiroptera: Pteropodidae). Mol Ecol. 2000;9:2198-201.

26. Matschiner $M$, Salzburger W. TANDEM: integrating automated allele binning into genetics and genomics workflows. Bioinformatics. 2009;25:1982-3.

27. Andrews S. FastQC: A quality control tool for high throughput sequence data. Reference Source. 2010.

28. Catchen J, Hohenlohe PA, Bassham S, Amores A, Cresko WA. Stacks: an analysis tool set for population genomics. Mol Ecol. 2013:22:3124-40.

29. Team RC. R: A language and environment for statistical computing. $R$ Foundation for Statistical Computing, Vienna, Austria. ISBN 3-900051-07-0, URL http://www.R-project.org/. 2014. 
30. Husson F, Josse J, Le S, Mazet J, Husson MF. Package 'FactoMineR'. 2014

31. Rousset F. Genepop'007: a complete re-implementation of the Genepop software for Windows and Linux. Mol Ecol Resour. 2008;8:103-6.

32. Goudet J. FSTAT, a program to estimate and test gene diversities and fixation indices (version 2.9. 3). 2001.

33. van Oosterhout C, Hutchinson WF, Wills DP, Shipley P. Micro-checker: software for identifying and correcting genotyping errors in microsatellite data. Mol Ecol Notes. 2004;4:535-8.

34. Foll M. BayeScan v2.1 User Manual. Ecology. 2012;20:1450-62.

35. Pritchard J, Wen X, Falush D. Documentation for STRUCTURE software: Version 2.3, Available from http://pritch.bsd.uchicago.edu/software. 2010.

36. Evanno G, Regnaut S, Goudet J. Detecting the number of clusters of individuals using the software STRUCTURE: a simulation study. Mol Ecol. 2005;14:2611-20.

37. Jakobsson M, Rosenberg NA. CLUMPP: a cluster matching and permutation program for dealing with label switching and multimodality in analysis of population structure. Bioinformatics. 2007;23:1801-6.

38. Nielsen EE, Bach LA, Kotlicki P. HYBRIDLAB (version 1.0): a program for generating simulated hybrids from population samples. Mol Ecol Notes. 2006;6:971-3.

39. Burgarella C, Lorenzo Z, Jabbour-Zahab R, Lumaret R, Guichoux E, Petit RJ, et al. Detection of hybrids in nature: application to oaks (Quercus suber and Q. ilex). Heredity. 2009;102:442-52

40. Kalinowski ST, Taper ML, Marshall TC. Revising how the computer program CERVUS accommodates genotyping error increases success in paternity assignment. Mol Ecol. 2007;16:1099-106.

41. Purcell S, Neale B, Todd-Brown K, Thomas L, Ferreira MAR, Bender D, et al. PLINK: a tool set for whole-genome association and population-based linkage analyses. Am J Hum Genet. 2007;81:559-75.

42. Ronquist F, Huelsenbeck JP. MrBayes 3. Bayesian phylogenetic inference under mixed models. Bioinformatics. 2003;19:1572-4.

43. Rambaut A, Drummond AJ. Tracer v. 1.5. Computer program and documentation distributed by the authors. 2007.

44. Rambaut A, Drummond AJ. FigTree. Program distributed by the authors. 2013.

45. Tamura K, Peterson D, Peterson N, Stecher G, Nei M, Kumar S. MEGA5: molecular evolutionary genetics analysis using maximum likelihood, evolutionary distance, and maximum parsimony methods. Mol Biol Evol. 2011:28:2731-9

46. Xia X, Xie Z. DAMBE: software package for data analysis in molecular biology and evolution. J Hered. 2001;92:371-3.

47. Eaton DAR. PyRAD: assembly of de novo RADseq loci for phylogenetic analyses. Bioinformatics. 2014; btu121.

48. Stamatakis A. RAxML version 8: a tool for phylogenetic analysis and postanalysis of large phylogenies. Bioinformatics. 2014;30:1312-3.

49. Durand $E Y$, Patterson N, Reich D, Slatkin M. Testing for ancient admixture between closely related populations. Mol Biol Evol. 2011;28:2239-52.

50. Eaton DAR, Ree RH. Inferring phylogeny and introgression using RADseq data: an example from flowering plants (Pedicularis: Orobanchaceae). Syst Biol. 2013;62:689-706.

51. Martin SH, Dasmahapatra KK, Nadeau NJ, Salazar C, Walters JR, Simpson F, et al. Genome-wide evidence for speciation with gene flow in Heliconius butterflies. Genome Res. 2013;23:1817-28.

52. Storz JF, Balasingh J, Bhat HR, Nathan PT, Doss D, Prakash AA, et al. Clinal variation in body size and sexual dimorphism in an Indian fruit bat, Cynopterus sphinx (Chiroptera: Pteropodidae). Biol J Linn Soc. 2001;72:17-31.

53. Storz JF, Beaumont MA. Testing for genetic evidence of population expansion and contraction: an empirical analysis of microsatellite DNA variation using a hierarchical Bayesian model. Evolution. 2002;56:154-66.

54. Berthier P, Excoffier L, Ruedi M. Recurrent replacement of mtDNA and cryptic hybridization between two sibling bat species Myotis myotis and Myotis blythii. P Roy Soc B- Biol Sci. 2006;273:3101-23.

55. Bogdanowicz W, Piksa K. Tereba A Genetic structure in three species of whiskered bats (genus Myotis) during swarming. J Mammal. 2012;93:799-807.

56. Larsen PA, Marchan-Rivadeneira M, Baker RJ. Natural hybridization generates mammalian lineage with species characteristics. Proc Natl Acad Sci U S A. 2010;107:11447-52.

57. Mao X, Zhang J, Zhang S, Rossiter SJ. Historical male-mediated introgression in horseshoe bats revealed by multilocus DNA sequence data. Mol Ecol. 2010;19:1352-66.
58. Huang $\mathrm{H}$, Knowles LL. Unforeseen consequences of excluding missing data from next-generation sequences: simulation study of RAD sequences. Syst Biol. 2014:syu046.

\section{Submit your next manuscript to BioMed Central and we will help you at every step:}

- We accept pre-submission inquiries

- Our selector tool helps you to find the most relevant journal

- We provide round the clock customer support

- Convenient online submission

- Thorough peer review

- Inclusion in PubMed and all major indexing services

- Maximum visibility for your research

Submit your manuscript at www.biomedcentral.com/submit
Biomed Central 